\title{
Avaliação do paciente em hemodiálise por meio da medida canadense de desempenho ocupacional ${ }^{*}$
}

\author{
Evaluation of the patient in hemodialysis by \\ canadian occupational performance measure
}

\author{
Crislaine Andolfato ${ }^{1}$, Milton Carlos Mariotti $^{2}$
}

\begin{abstract}
ANDOLFATO, C.; MARIOTTI, M. C. Avaliação do paciente em hemodiálise por meio da medida canadense de desempenho ocupacional. Rev. Ter. Ocup. Univ. São Paulo, v. 20, n. 1, p.1-7, jan./ abr. 2009.

RESUMO: O portador de insuficiência renal crônica (IRC) em hemodiálise sofre diversas alterações em sua rotina diária, havendo a necessidade do desenvolvimento de estratégias capazes de auxiliálo durante este processo. OBJETIVO: o objetivo foi avaliar a mudança ocorrida na autopercepção do portador de IRC em hemodiálise sobre seu desempenho ocupacional, após a intervenção da Terapia Ocupacional. MÉTODO: participaram do estudo 21 portadores de IRC em hemodiálise, atendidos na Clínica de Doenças Renais, localizada em Curitiba, PR. Os sujeitos foram avaliados por meio da Medida Canadense de Desempenho Ocupacional - COPM e reavaliados após período de intervenção da Terapia Ocupacional, a qual foi baseada no Modelo Canadense de Desempenho Ocupacional. RESULTADOS: os maiores problemas de desempenho ocupacional apresentados pelos sujeitos foram controlar a dieta líquida e sólida, participar de atividades sociais e freqüentar cursos. O percentual de sujeitos que apresentou variação de 2 ou mais pontos nos escores de satisfação e desempenho foi de $52,4 \%$ e $47,6 \%$, respectivamente. A COPM foi capaz de mensurar os problemas existentes no desempenho ocupacional do portador de IRC em hemodiálise e principalmente, as mudanças ocorridas após a intervenção da Terapia Ocupacional.
\end{abstract}

DESCRITORES: Terapia ocupacional. Diálise renal. Insuficiência renal crônica. Avaliação em saúde.

\footnotetext{
*Trabalho desenvolvido durante o programa de Iniciação Científica 2008/2009 do Curso de Terapia Ocupacional da UFPR. Apresentado no $17^{\circ}$ Evento de Iniciação Científica (EVINCI) da UFPR. Recebeu apoio na modalidade de bolsa de Iniciação Científica pela UFPR/ TN - Edital IC 2008-2009.

1. Acadêmica do $9^{\circ}$ período do curso de graduação de Terapia Ocupacional e Bolsista de Iniciação Científica pela UFPR. e-mail: crislaineandolfato@hotmail.com.

${ }^{2}$. Terapeuta Ocupacional, Mestre em Educação, Professor Assistente do curso de graduação de Terapia Ocupacional da UFPR. e-mail: mariotti@ufpr.br

Endereço para correspondência: Rua Padre Camargo, 280 - $3^{\circ}$ andar. Bairro Alto da Glória, Curitiba, PR. CEP: 80060-240
} 


\section{INTRODUÇÃO}

$\mathrm{A}$ Insuficiência Renal Crônica (IRC) caracterizase pela lesão, com perda progressiva e irreversível da função dos rins. Devido a capacidade de adaptação destes órgãos à perda crônica de néfrons, a homeostase do organismo é mantida até fases avançadas da doença, fazendo com que o indivíduo permaneça assintomático durante vários anos (ROMÃO Jr, 2004; ZATZ, 2003). Quando os rins não conseguem mais eliminar as toxinas liberadas pelo metabolismo e manter a composição físico-química do organismo dentro da normalidade, é necessário submeter o doente ao tratamento de substituição renal (ZATZ, 2003).

Um dos tratamentos utilizados na doença renal crônica é a hemodiálise, a qual substitui a função dos rins pelo processo de circulação sanguínea extracorpórea, removendo as substâncias nocivas ao organismo. O tratamento hemodialítico é realizado 3 vezes por semana com cada sessão durando entre 3 e 4 horas (LUGON et al., 2003; TRENTINI et al., 2004).

A Sociedade Brasileira de Nefrologia (SBN) estimou que 87.044 pessoas se encontravam em tratamento dialítico no Brasil em março de 2008 (prevalência de 468/1.000.000). $\mathrm{O}$ crescimento esperado no número de pessoas em diálise para o mesmo ano era de $18,3 \%$ em relação a 2007 . Em 2007, 66.833 pessoas submetidas a diálise crônica faziam tratamento de hemodiálise e 6.772 de diálise peritoneal (SESSO et al., 2007, 2008). Romão Jr (2006), afirma que a doença renal crônica constitui-se em um importante problema de saúde pública no Brasil.

Preocupados com o cotidiano e com a qualidade de vida do portador de IRC em hemodiálise, terapeutas ocupacionais desenvolveram estudos que avaliaram a percepção sobre o funcionamento ocupacional (BEZERRA; SANTOS, 2008) e sobre o impacto de um programa de Terapia Ocupacional na qualidade de vida destas pessoas (MARIOTTI; CARVALHO, 2007). Observou-se a necessidade do desenvolvimento de estudos que verificassem quais são os problemas de desempenho ocupacional apresentados por esta população, assim como, estratégias de intervenção capazes de auxiliá-la. Os autores acima afirmam que a vida cotidiana do doente renal crônico em hemodiálise sofre modificações e que a Terapia Ocupacional pode contribuir para a sua reestruturação.

Como o desempenho ocupacional, foco de intervenção da Terapia Ocupacional, é determinado pelo indivíduo com base em suas experiências, ninguém melhor do que ele para determinar quais habilidades possui para desempenhar suas ocupações diárias, assim como, a satisfação que tem com este desempenho (LAW et al., 2009). Sendo a Medida Canadense de Desempenho Ocupacional (Canadian Occupational Performance Measure - COPM), um instrumento capaz de avaliar estes parâmetros, ela foi utilizada neste estudo.

Law et al. (2009) afirmam que a COPM é uma medida de resultados padronizada em forma de entrevista semi-estruturada, utilizada por terapeutas ocupacionais em pessoas com vários tipos de incapacidades e em todos os estágios de desenvolvimento, a fim de detectar mudanças na autopercepção do cliente sobre seu desempenho ocupacional ao longo do tempo.

Publicada pela primeira vez em 1990, a COPM foi traduzida oficialmente para 24 idiomas e é utilizada em mais de 35 países, sendo que até o momento, mais de 80 trabalhos investigaram o seu uso, incluindo a avaliação da sua capacidade psicométrica, confiabilidade, validade e utilidade (McCOLL et al., 2005).

Embora não tenha sido publicado, o formulário da COPM foi traduzido para o português em 1.999 pela professora Silvia Gonçalves, da Escola Superior de Saúde do Alcoitão, em Lisboa, Portugal. O Manual de Instrução foi preparado na versão brasileira e publicado em 2009 por Lívia C. Magalhães, Lilian V. Magalhães e Ana Amélia Cardoso (MAGALHÃES et al., 2009). No entanto até o momento não foi encontrada literatura descrevendo a utilização do referido instrumento de avaliação com portadores de IRC em hemodiálise.

Este estudo trata de uma pesquisa qualiquantitativa desenvolvida a partir de um Projeto de Iniciação Científica realizado nos anos de 2008-2009, na Universidade Federal do Paraná (UFPR). O objetivo foi avaliar a mudança ocorrida na autopercepção do portador de IRC em hemodiálise sobre seu desempenho ocupacional, após a intervenção da Terapia Ocupacional. Para tanto, utilizouse um instrumento de avaliação específico de Terapia Ocupacional que pudesse mostrar quais são as dificuldades no desempenho ocupacional, apresentadas por portadores de IRC em hemodiálise.

\section{PROCEDIMENTOS E MÉTODOS}

\section{Participantes}

São sujeitos deste estudo, portadores de IRC que se encontravam em tratamento em uma Clínica de Doenças Renais, em Curitiba, no período de agosto a novembro de 2008. A clínica possui equipe multidisciplinar composta por médicos, enfermeiros, técnicos em enfermagem, psicólogos, assistentes sociais e nutricionistas e atende em média 260 pessoas oriundas da cidade de Curitiba e região 
metropolitana. O serviço de Terapia Ocupacional existe pelo convênio estabelecido entre a clínica e o curso de graduação de Terapia Ocupacional da UFPR.

Os critérios para inclusão dos sujeitos no estudo foram que estes estivessem com estado clínico estável, fossem adultos ou idosos, portadores de IRC em tratamento de hemodiálise na clínica, não possuíssem atividade profissional naquele momento e manifestassem interesse em participar do atendimento de Terapia Ocupacional. Foram excluídos os sujeitos que não atendiam aos critérios de inclusão e que não apresentavam condições cognitivas para compreender o instrumento de avaliação utilizado (COPM).

Inicialmente foram incluídos 35 sujeitos. No decorrer do estudo, 4 realizaram transplante renal, 6 desistiram de participar da pesquisa devido ao estado de saúde debilitado, 1 foi a óbito, 2 não apresentaram problemas de desempenho ocupacional e 1 passou a realizar diálise peritoneal, portanto, concluíram a pesquisa 21 sujeitos.

Os participantes do estudo assinaram termo de consentimento informado e a pesquisa foi apreciada pelo comitê de Ética do Setor de Ciências da Saúde da Universidade Federal do Paraná.

\section{Aplicação da COPM e intervenção da terapia ocupacional}

Tanto as avaliações iniciais e finais, quanto as intervenções, ocorreram enquanto os clientes realizavam a hemodiálise e foram desenvolvidas por 3 acadêmicas do $8^{\circ}$ período de Terapia Ocupacional que participavam do Programa de Iniciação Científica do curso de Graduação de Terapia Ocupacional da Universidade Federal do Paraná . O tempo médio para a avaliação inicial variou entre 20-40 minutos e para a reavaliação de 5-10 minutos.

O primeiro passo para a avaliação inicial foi a entrevista sobre o desempenho ocupacional. Nesta, os sujeitos puderam identificar as atividades incluídas nas três áreas de desempenho ocupacional (autocuidado, produtividade e lazer), que eles gostariam de realizar, que precisavam ou que era esperado que realizassem. Depois disso foi perguntado se eram capazes de realizar estas atividades e se estavam satisfeitos com a maneira como as realizavam (o protocolo da COPM traz exemplos de atividades que podem constar em cada área de desempenho, no entanto, como indicado no manual, a avaliação inicial não se restringiu apenas a estes exemplos). Cada sujeito pôde apontar até 5 problemas de desempenho ocupacional que considerava mais importante, os quais compuseram os objetivos do processo de intervenção da Terapia Ocupacional.

Para cada problema identificado, puderam pontuar o grau de desempenho e satisfação numa escala numérica de 1 a 10. Para avaliar o desempenho foi feita a seguinte pergunta: "Como você pontuaria a maneira como você realiza esta atividade agora?" (LAW et al., 2009, p. 28). Quanto a avaliação da satisfação, foi perguntado: "O quanto você está satisfeito com a maneira que você realiza esta atividade agora?" (LAW et al., 2009, p. 29).

O próximo passo foi quantificar desempenho e satisfação separadamente. Para isto, foram somadas as pontuações obtidas com o desempenho e depois, o valor foi dividido pelo número de problemas, gerando desta forma, o escore total do desempenho. O mesmo procedimento foi efetuado para obtenção do escore de satisfação.

Concluída a avaliação inicial foi elaborado o plano de intervenção baseado nas queixas referidas pelos sujeitos. A intervenção foi baseada no Modelo Canadense de Desempenho Ocupacional (MCDO). Este modelo é centrado no cliente e tem como pressuposto que o desempenho ocupacional é resultado da interação entre a pessoa, o ambiente e a ocupação (HAGEDORN, 2001; LAW et al., 2009).

$\mathrm{Na}$ reavaliação, após 16 sessões de Terapia Ocupacional, incluindo a primeira avaliação, os sujeitos foram novamente questionados sobre o grau de desempenho e satisfação em cada atividade pontuada na avaliação inicial. Para averiguar a variação (em pontos) nos escores de desempenho e satisfação de cada sujeito, realizou-se o seguinte cálculo, conforme o manual da COPM (LAW et al., 2009): o desempenho inicial foi subtraído do desempenho final, gerando o escore para desempenho; e a satisfação inicial foi subtraída da satisfação final, gerando o escore para satisfação.

Os dados estatísticos foram calculados por meio do programa computadorizado Microsoft Office Excel 2007.

\section{RESULTADOS}

Dos 21 sujeitos da pesquisa, 11 eram do gênero masculino (52,4\%) e 10 do feminino (47,6\%). A prevalência foi de pessoas casadas ou com união estável $(71,4 \%)$ e $1^{\circ}$ grau incompleto (52,4\%). A faixa etária variou entre 21 e 72 anos $(<20=0 \%$, entre $20-39=23,8 \%$, entre $40-60=52,4 \%,>60$ $=23,8 \%)$. O tempo de tratamento em hemodiálise oscilou entre 1 a 17 anos e o de permanência na máquina, entre $3 \mathrm{~h}$ e $4 \mathrm{~h}$, sendo que todos realizavam tal procedimento 3 vezes por semana e estavam aposentados ou recebendo auxílio doença 
pelo Instituto Nacional da Seguridade Social (INSS).

O tempo dispendido para a avaliação inicial ficou entre 20-40 minutos e para a reavaliação entre 5-10 minutos.
Os principais problemas encontrados no desempenho ocupacional dos sujeitos da pesquisa estão relacionados na Tabela 1.

TABELA 1. Problemas de desempenho apontados pelos sujeitos

\begin{tabular}{lcc}
\hline ATIVIDADES & & TOTAL \\
\hline & $\mathrm{n}^{\circ}$ & $\%$ \\
Controlar a dieta líquida e sólida & 07 & 9,7 \\
Participar de atividades sociais & 06 & 8,3 \\
Frequentar cursos & 06 & 8,3 \\
Praticar hobbies & 05 & 6,9 \\
Realizar limpezas & 05 & 6,9 \\
Realizar atividades voluntárias & 05 & 6,9 \\
Praticar esportes & 04 & 5,6 \\
Frequentar escola & 04 & 5,6 \\
Tomar remédios & 04 & 5,6 \\
Deambular fora de casa & 03 & 4,2 \\
Utilizar onnibus & 03 & 4,2 \\
Lavar roupas & 03 & 4,2 \\
Fazer artesanato & 02 & 2,8 \\
Realizar visitas & 02 & 2,8 \\
Viajar & 02 & 2,8 \\
Deambular dentro de casa & 02 & 2,8 \\
Retornar ao trabalho & 02 & 2,8 \\
Banhar-se & 01 & 1,4 \\
Fazer compras & 01 & 1,4 \\
Cortar grama & 01 & 1,4 \\
Manter-se no emprego & 01 & 1,4 \\
Cortar alimentos (preparo de refeições) & 01 & 1,4 \\
Receber auxílio doença & 01 & 1,4 \\
Realizar passeios & 01 & 1,4 \\
\hline
\end{tabular}

$\mathrm{n}^{\mathrm{o}}=$ número de vezes em que a atividade foi citada

Após 16 sessões de Terapia Ocupacional, foi realizada a reavaliação. Tendo como base a avaliação inicial verificouse que houve um percentual de sujeitos que não sofreu alteração no desempenho e satisfação, outro que diminuiu o escore enquanto que o maior percentual variou positivamente (Gráfico 1). O percentual de sujeitos que apresentou variação de 2 ou mais pontos nos escores de satisfação e desempenho foi de $52,4 \%$ e $47,6 \%$, respectivamente.

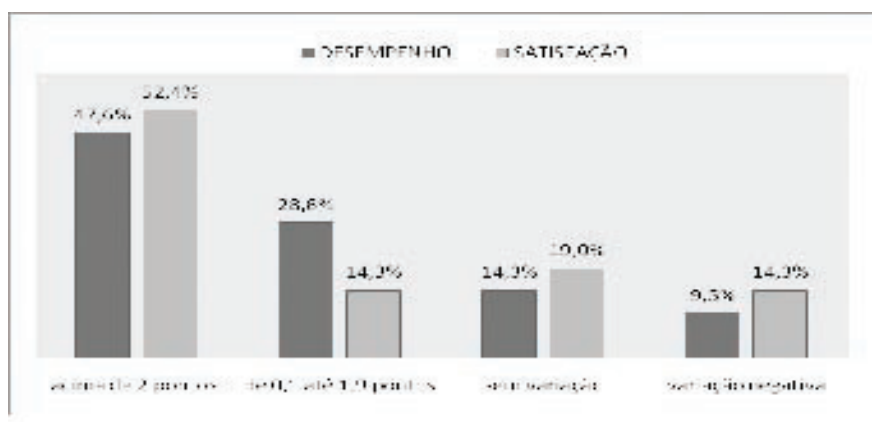

GRÁFICO 1. Variação dos escores 


\section{DISCUSSÃO}

Para Bezerra e Santos (2008), devido aos sinais, sintomas e tratamento da doença, o portador de IRC sofre uma série de limitações físicas, sociais e emocionais, incluindo dificuldades no desempenho ocupacional, restrições hídricas, dietas especiais, consultas médicas e sessões de hemodiálise, tornando a pessoa frágil e desestruturando o seu cotidiano.

No total foram 24 os problemas de desempenho ocupacional apontados pelos sujeitos da pesquisa, sendo que as maiores dificuldades estavam em controlar a dieta líquida e sólida, participar de atividades sociais e frequentar cursos. Analisando todas as atividades citadas, os resultados sugerem que as limitações ocasionadas pela IRC geravam nestes sujeitos, dificuldades que influenciavam sua rotina diária, o contato social e a permanência no trabalho, enfim, comprometia seu desempenho ocupacional tanto nas atividades de autocuidado como nas atividades produtivas e de lazer.

Quanto aos escores obtidos na reavaliação após intervenção da Terapia Ocupacional, constatou-se que houve variação acima de 2 pontos para satisfação e desempenho em $52,4 \%$ e $47,6 \%$ dos sujeitos respectivamente. Variação de 2 ou mais pontos na COPM é considerado clinicamente significativo (CARSWELL, 2004; LAW et al., 2009). Houve uma parcela que sofreu variação nos escores de desempenho e satisfação, entre 0,1 e 1,9 pontos $(14,3 \%$ para satisfação e $28,6 \%$ para desempenho). As variações positivas nos escores podem ser entendidas a partir de três condições: a intervenção da Terapia Ocupacional foi eficiente, o uso da COPM possibilitou o maior engajamento do sujeito no processo terapêutico, como afirmam Law et al. (2009), ou, o seu estado geral de saúde melhorou.

Para um percentual dos sujeitos pesquisados não houve variação nos escores de satisfação e desempenho, 19,0\% e $14,3 \%$ respectivamente. A variação do escore para satisfação foi negativa em $14,3 \%$, enquanto que para desempenho, em $9,5 \%$ dos sujeitos. Law et al. (2009), relatam que um aumento no nível de discernimento pode levar o indivíduo a pontuar seu desempenho e satisfação mais baixo na reavaliação. No entanto, não é possível afirmar se as variações negativas nos escores foram resultado de falhas na intervenção, se houve aumento da percepção do sujeito sobre os déficits em seu desempenho ocupacional, ou ainda, deterioração no seu estado de saúde, ocasionando desta forma, piora em seu desempenho ocupacional e consequente insatisfação com o mesmo.

Não foi realizada comparação de desempenho entre sujeitos, pois, a COPM possui um sistema estruturado de pontuação e seus valores são dependentes dos problemas identificados por cada sujeito. Desta forma é difícil comparar e interpretar diferenças de escores entre indivíduos. ACOPM não foi desenvolvida para avaliar desvios no desempenho ocupacional a partir de normas ou critérios de normalidade derivados empiricamente, já que esta avaliação não impõe padrão para desempenho ocupacional baseado na população em geral, assim, os escores obtidos com cada sujeito são comparados com ele mesmo (avaliação inicial e final). Isto porque, a base teórica sobre a qual a medida foi desenvolvida descreve o desempenho ocupacional como uma experiência individual subjetiva, afirmam Law et al. (2009).

Nem todos os problemas relatados pelos sujeitos puderam ser solucionados da forma como eles esperavam, como exemplos estão as atividades: manter-se no emprego e retornar ao trabalho. Elas foram mantidas como objetivos da intervenção por ter sido utilizada a abordagem centrada no cliente. Como não havia possibilidade da manutenção do emprego e do retorno ao trabalho devido aos fatores que envolvem a IRC, isto foi trabalhado durante os atendimentos de Terapia Ocupacional, buscando-se a conscientização dos envolvidos de que naquele momento, não era possível alcançar estes objetivos.

O tempo utilizado para a avaliação inicial foi de 20-40 minutos, portanto, acima daquele mencionado por Law et al. (2009) que afirmam que a avaliação não deve ultrapassar 15-30 minutos. O tempo superior ao estipulado pelos autores justifica-se pelas condições em que a COPM foi aplicada nesta pesquisa: as sessões de hemodiálise foram seguidas por interrupções freqüentes da equipe de saúde a fim de aferir pressão arterial, coletar assinatura em prontuário, administrar medicações, regular os dispositivos da máquina de hemodiálise. Além disso, alguns sujeitos não compreenderam o significado da palavra "desempenho", sendo necessário realizar explicações baseadas em exemplos.

Os dados descritos por este estudo não foram comparados a investigações similares, por estas não terem sido encontradas na literatura.

\section{CONCLUSÃO}

Verificou-se que as dificuldades presentes no desempenho ocupacional dos sujeitos desta pesquisa, corroboram aquelas descritas pela literatura, mostrando que a COPM é um instrumento de avaliação que pode ser utilizado por terapeutas ocupacionais com esta clientela, pois conseguiu mensurar as dificuldades no desempenho ocupacional do portador de IRC em hemodiálise e, principalmente, as mudanças ocorridas neste desempenho 
após a intervenção da Terapia Ocupacional.

$\mathrm{O}$ uso da abordagem centrada no cliente, modelo teórico sob a qual a COPM foi desenvolvida, permitiu maior envolvimento dos sujeitos no processo terapêutico ocupacional, possibilitando que os objetivos traçados inicialmente fossem em grande parte alcançados, embora as condições para os atendimentos fossem pouco favoráveis, por terem sido realizados durante as sessões de hemodiálise.

Observa-se a necessidade de que novas pesquisas sejam desenvolvidas a fim de que estes dados possam ser comparados, já que não foram encontrados outros estudos envolvendo o uso da COPM com portadores de IRC em hemodiálise.

AGRADECIMENTOs: Agradecemos as acadêmicas, bolsistas de iniciação científica do Curso de Terapia Ocupacional da UFPR, Andressa Santi e Bruna Doubeck Cavet Javorouski, por suas contribuições para o desenvolvimento deste estudo.

ANDOLFATO, C.; MARIOTTI, M. C. Evaluation of the patient in hemodialysis by Canadian Occupational Performance Measure. Rev. Ter. Ocup. Univ. São Paulo, v. 20, n. 1, p. 1-7, jan./ abr. 2009.

\begin{abstract}
The chronic kidney patient under hemodialysis undergoes several changes in his/her daily routine, with the need for strategies able to assist him/her during this process. OBJECTIVE: The objective of the study was to evaluate the changes occurred in the self-perception of the chronic kidney patients under hemodialysis as regards his/her occupational performance, after the occupational therapy intervention. METHOD: participants were 21 patients with chronic kidney patient under hemodialysis, treated at a Renal Diseases Clinic, located in Curitiba, PR. Subjects were assessed using the Canadian Occupational Performance Measure - COPM and reassessed after the intervention period of Occupational Therapy, which was based on the Canadian Occupational Performance Model. RESULTS: the major problems found in the occupational performance of the subjects were to control the solid and liquid diet and to participate in social activities and courses. The percentage of subjects that vary from 2 or more points in scores for satisfaction and performance was $52.4 \%$ and $47.6 \%$, respectively. The COPM was able to measure problems in occupational performance of the bearer of chronic kidney patient under hemodialysis and mainly the changes after the intervention of Occupational Therapy.
\end{abstract}

KEY WORDS: Occupational therapy. Renal dialysis. Renal insufficiency, chronic. Health evaluation.

\title{
REFERÊNCIAS
}

BEZERRA, K. V.; SANTOS, J. L. F. Daily life of patients with chronic renal failure receiving hemodialysis treatment. Rev. Latinoam. Enferm. [online], v. 16, n. 4, p. 686-691, jul./ago. 2008. Disponível em: <http://www.scielo.br/pdf/rlae/v16n4/pt_06.pdf >. Acesso em: 11 fev. 2009.

CARSWELL, A.; MCCOLL, M. A.; BAPTISTE, S.; LAW, M.; POLATAJKO, H. L.; POLLOCK, N. The Canadian occupational performance measure: a research and clinical literature review. Can. J. Occup. Ther., v. 71, n. 4, p. 210-222, 2004.

HAGEDORN, R. Fundamentos da prática em terapia ocupacional. São Paulo: Dinamys Editorial, 2001.
LAW, M.; BAPTISTE, S.; CARSWELL, A.; McCOLL, M. A.; POLATAJKO, H. L.; POLLOCK, N. Medida Canadense de Desempenho Ocupacional (COPM). Tradução e organização de Lívia de Castro Magalhães, Lilian Vieira Magalhães e Ana Amélia Cardoso. Belo Horizonte: Editora Universidade Federal de Minas Gerais, 2009.

LUGON, J. R.; MATOS, J. P. S.; WARRAK, E. A. Hemodiálise. In: RIELLA, M. C. Princípios de nefrologia e distúrbios hidroeletroliticos. 4. ed. Rio de Janeiro: Guanabara Koogan, 2003. p. 869-907.

MAGAlHÃES, L.C.; MAGALHÃES, L. V.; CARDOSO, A. 
A. Apresentação. In: LAW, M.; BAPTISTE, S.; CARSWELL, A.; McCOLL, M. A.; POLATAJKO, H.; POLLOCK, N. Medida Canadense de Desempenho Ocupacional (COPM). Tradução e organização de Lívia de Castro Magalhães, Lilian Vieira Magalhães e Ana Amélia Cardoso. Belo Horizonte: Editora Universidade Federal de Minas Gerais, 2009. p. 9.

MARIOTTI, M. C.; CARVALHO, J. G. R. Avaliação do impacto de um programa de terapia ocupacional na qualidade de vida do portador de insuficiência renal crônica em hemodiálise: estudo piloto. In: CONGRESSO BRASILEIRO DE TERAPIA OCUPACIONAL, 10., Goiânia, 2007. Anais em CD. Goiânia: Associação dos Terapeutas Ocupacionais de Goiás, 2007.

McCOLL, M. A.; LAW, M.; BAPTISTE, S.; POLLOCK, N.; CARSWELL, A.; POLATAJKO, H.L. Targeted applications of the Canadian occupational performance measure. Can. J. Occup. Ther, v. 72, n. 5, p. 298-300, 2005.

ROMÃO Jr, J. E. Doença renal crônica: definição, epidemiologia e classificação. J. Bras. Nefrol., v. 26, n. 3, supl. 1, p. 1-3, 2004.
ROMÃO Jr, J. E. Insuficiência renal crônica. In: CRUZ, J.; PRAXEDES, J. N.; CRUZ, H. M. M. Nefrologia. 2. ed. São Paulo: Sarvier, 2006. p. 248-265.

SESSO, R.; LOPES, A. A.; THOMÉ, F. S.; BEVILACQUA, J. L.; ROMÃO Jr, J. E.; LUGON, J. Resultado do censo de diálise da SBN, 2007. J. Bras. Nefrol., v. 29, n. 4, p. 197-202, 2007.

SESSO, R.; LOPES, A. A.; THOMÉ, F. S.; BEVILACQUA, J. L.; ROMÃO Jr, J. E.; LUGON, J. Relatório do censo de diálise da SBN, 2008. J. Bras. Nefrol., v. 30, n. 4, p. 233-238, 2008.

TRENTINI, M.; CORRADI, E. M.; ARALDI, M. A. R.; TIGRINHO, F. C. Qualidade de vida de pessoas dependentes de hemodiálise considerando alguns aspectos físicos, sociais e emocionais. Rev. Texto Contexto Enferm., v. 13, n. 1, p. 74-82, 2004.

ZATZ, R. Insuficiência renal crônica (IRC). In: RIELLA, M. C. Principios de nefrologia e distúrbios hidroeletrolíticos. 4. ed. Rio de Janeiro: Guanabara Koogan, 2003. p. 649-60. 